\title{
CANONICALLY FIBERED SURFACES OF GENERAL TYPE
}

\author{
XIN LÜ† \\ Department of Mathematics, Shanghai Key Laboratory of PMMP, \\ East China Normal University, Dongchuan RD 500, Shanghai 200241, PR China \\ (x.lu@uni-mainz.de)
}

(Received 5 September 2016; revised 29 November 2017; accepted 10 December 2017; first published online 18 January 2018)

In memory of my father

Abstract In this paper, we construct the first examples of complex surfaces of general type with arbitrarily large geometric genus whose canonical maps induce non-hyperelliptic fibrations of genus $g=4$, and on the other hand, we prove that there is no complex surface of general type whose canonical map induces a hyperelliptic fibrations of genus $g \geqslant 4$ if the geometric genus is large.

Keywords: canonical map; canonically fibered surface; hyperelliptic fibration; non-hyperelliptic fibration 2010 Mathematics subject classification: 14J25; 14J29

\section{Contents}

3 Non-hyperelliptic canonical fibration of genus $g=4 \quad 214$

3.1 The construction . . . . . . . . . . . . . . . . . 214

3.2 The invariants . . . . . . . . . . . . . . . 216

4 Hyperelliptic canonical fibration $\quad 219$

4.1 The case of elliptic base . . . . . . . . . . . . . . . . . 219

4.2 The case of rational base . . . . . . . . . . . . . . . . 221

References

This work is supported by SFB/Transregio 45 Periods, Moduli Spaces and Arithmetic of Algebraic Varieties of the Deutsche Forschungsgemeinschaft (DFG), and partially supported by NSFC.

†Current address: Institut für Mathematik, Universität Mainz, Mainz, Germany, 55099. 


\section{Introduction}

Let $S$ be a smooth projective surface of general type over $\mathbb{C}$ and $K_{S}$ its canonical divisor. Denote by $\phi_{n}$ the map determined by the complete linear system $\left|n K_{S}\right|$. We call $\phi_{1}$ the canonical map, and $\phi_{n}(n \geqslant 2)$ the pluri-canonical maps of $S$.

Much progress has been made in the study of the pluri-canonical maps; see for instance, Reider's method [15]. However, the case of canonical map is different. Most of the methods used in the study of the pluri-canonical maps are no longer valid. In [2], Beauville first studied the canonical map systematically.

In this paper, we are mainly interested in the case when the image of the canonical map is a curve, in which case we say that $S$ admits a canonical fibration or $S$ is a canonically fibered surface. In fact, by blowing up the possible base points of the rational map $\phi_{1}$ and the Stein factorization, one obtains a fibration $f: X \rightarrow B$, which is called a canonical fibration following Sun [17]. Denote by $g$ the genus of a general fiber.

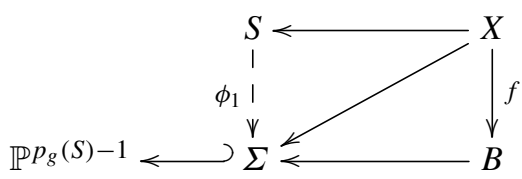

The following result was established in [2].

Theorem 1.1 (Beauville). If $S$ admits a canonical fibration of genus $g$ and $\chi\left(\mathcal{O}_{S}\right) \geqslant 21$, then $2 \leqslant g \leqslant 5$, and

$$
K_{S}^{2} \geqslant 3 p_{g}(S)-6 .
$$

In the same paper, Beauville also constructed examples of canonically fibered surfaces with $g=2$ and 3, where the geometric genus can be arbitrarily large. See also [3, 14, 19, $20,25,26]$ for such examples. We also refer to [13] for a systematical study of canonically fibered surfaces with $g=2$. In [20], Xiao improved the above inequality in the case when $g=2$ (with one exception where $S$ is minimal with $K_{S}^{2}=1$ and $p_{g}(S)=2$ ):

$$
K_{S}^{2} \geqslant 4 p_{g}(S)-6, \quad \text { if } g=2 .
$$

In fact, the above inequality holds for all canonically fibered surfaces, cf. [23, 24]. The following questions arise naturally (cf. [21, Problem 6]).

(1) Does there exist any surface of general type with a canonical fibration of genus $g=4$ or 5 with arbitrarily high geometric genus.

(2) When $S$ admits a canonical fibration of genus $g$, find the largest $c(g)$ such that $K_{S}^{2} \geqslant c(g) \cdot p_{g}(S)+$ some constant.

As mentioned above, Xiao proved that $c(2)=4$. Beauville showed in [2] that $c(g) \geqslant$ $2 g-2$. More precisely, he proved

$$
K_{S}^{2} \geqslant(2 g-2)\left(p_{g}(S)-1\right)
$$

In $[16,17]$, Sun proved that 
Theorem 1.2 (Sun). (i) If $S$ admits a canonical fibration of genus $g$ and $p_{g}(S)>2 g-$ 2 , then

$$
K_{S}^{2} \geqslant\left(2 g-2+\frac{2 g-2}{2 g-1}\right)\left(p_{g}(S)-1\right)-\frac{8(g-1)^{2}}{2 g-1} .
$$

(ii) If $S$ admits a canonical fibration of genus $g=3$, then

$$
K_{S}^{2} \geqslant \frac{21}{4} p_{g}(S)-\frac{71}{6}
$$

(iii) If $S$ admits a canonical fibration of genus $g$ which is hyperelliptic, then

$$
K_{S}^{2} \geqslant \begin{cases}2\left(g-\frac{1}{g}\right)\left(p_{g}(S)+1\right)-8(g-1) & \text { if } g(B)=0, \\ 2\left(g-\frac{1}{g}\right) p_{g}(S) & \text { if } g(B)=1 .\end{cases}
$$

In particular, there is no surface of general type admitting a hyperelliptic canonical fibration of $g=5$ if either $g(B)=1$ or $p_{g}(S) \geqslant 53-15 q(S)$.

Very recently, Chen [5] studied the non-hyperelliptic canonical fibration of genus 5, which together with Sun's result confirms that there is no surface of general type with a canonical fibration of genus $g=5$ if either $g(B)=1$ or $p_{g}(S) \geqslant 1952$. However, it has been always mysterious whether there exists a surface of general type with a canonical fibration of genus $g=4$ or not.

Our main results are the following.

Theorem 1.3. (i) There exist a sequence of surfaces of general type with arbitrarily large geometric genus which admit non-hyperelliptic canonical fibrations of genus $g=4$.

(ii) There exists no surface of general type which admits a hyperelliptic canonical fibration of genus $g \geqslant 4$ if either $g(B)=1$ or $p_{g}(S) \geqslant 212-72 q(S)$.

Remarks 1.4. (i) Together with Chen's result [5], our examples settle the question of which genera can appear in a canonical fibration.

(ii) It is not difficult to construct surfaces of general type which admit hyperelliptic canonical fibrations of genus $g=2$ or 3 as remarked above; see also Example 4.10. Hence the bound on the genus appearing in a hyperelliptic canonical fibration is sharp.

Outline of the exclusion of hyperelliptic canonical fibrations of genus $g \geqslant 4$. Let $S$ be a surface admitting a hyperelliptic canonical fibration, and $f: X \rightarrow B$ be the induced fibration of curves of genus $g \geqslant 2$. It is well known that the base $B$ is either elliptic or rational. The technique differs in these two cases.

In the case when $B$ is elliptic, the relative canonical divisor $K_{X / B}=K_{X}$. Hence one deduces a decomposition (announced in [7], and a complete proof can be found in the recent paper [4])

$$
f_{*} \mathcal{O}_{X}\left(K_{X}\right)=\mathcal{L} \oplus \mathcal{F}
$$


where $\mathcal{L}$ is an ample line bundle on $B$ with $h^{0}(\mathcal{L})=p_{g}(S)$, and $\mathcal{F}$ is a unitary subbundle on $B$ with $\operatorname{rank} \mathcal{F}=g-1$. We will show that, up to a suitable étale base change, $\mathcal{F}$ becomes trivial (cf. Proposition 4.1). Together with the slope inequality for hyperelliptic fibrations with positive relative irregularity (cf. [9, Theorem 1.4]), we prove that

Theorem 1.5. Assume that $S$ admits a hyperelliptic canonical fibration of genus $g$. If the base $B$ is elliptic, then $g \leqslant 3$, and

$$
K_{S}^{2} \geqslant \begin{cases}4 p_{g}(S) & \text { if } g=2 \\ 8 p_{g}(S) & \text { if } g=3\end{cases}
$$

If the base $B$ is rational, then we do not have such a decomposition for $f_{*} \mathcal{O}_{X}\left(K_{X}\right)$, and the above technique does not work any more. Write

$$
K_{X}=Z+M=D+V+M
$$

where $D$ (resp. $V$ ) is the horizontal (resp. vertical) part of the fixed part $Z$ contained in $\left|K_{X}\right|$, and $M$ is the moving part. It is clear that $M \equiv\left(p_{g}-1\right) F$, where $F$ is a general fiber of $f$. To obtain a lower bound on $K_{S}^{2}$, it suffices to bound the canonical degree of $D$, i.e., the intersection $K_{X} D$, from below. This will be done by applying the hyperelliptic involution on $X$ together with the Riemann-Roch theorem on the associated ruled surface (cf. Lemmas 4.5 and 4.7). As a consequence, we show that

Theorem 1.6. Assume that $S$ admits a hyperelliptic canonical fibration of genus $g$. If the base $B \cong \mathbb{P}^{1}$, then

$$
K_{S}^{2} \geqslant 3(g-1)\left(p_{g}(S)-2\right)
$$

Remarks 1.7. (i) The idea of bounding the canonical degree of $D$ using the hyperelliptic involution on $X$ and the Riemann-Roch theorem on the associated ruled surface goes back to Sun [16, 17]. In fact, Sun has studied in [16] the case when the horizontal fixed part $D=(2 g-2) C$ with $C$ being a section of $f$, and obtained the same bound (1.2) in this special case.

(ii) The inequality (1.2) together with (1.1) improves Sun's bound in the case when $S$ admits a hyperelliptic canonical fibration of genus $g=3$. When $g=2$, (1.1) has already been proved by Xiao [20]; while (1.2) is weaker than Xiao's.

Combining the above bound together with the logarithmic type Miyaoka-Yau inequality for surfaces of general type, we exclude the existence of a surface of general type with a hyperelliptic canonical fibration over $\mathbb{P}^{1}$ of genus $g \geqslant 4$.

Organization. Our paper is organized as follows. In $\S 2$, we do some preliminaries and introduce the notations. In $\S 3$, we construct a sequence of surfaces of general type which admit non-hyperelliptic fibrations of genus $g=4$. In $\S 4$, we exclude the existence of surfaces of general type with a hyperelliptic canonical fibration of genus $g \geqslant 4$ along the idea illustrated above. 


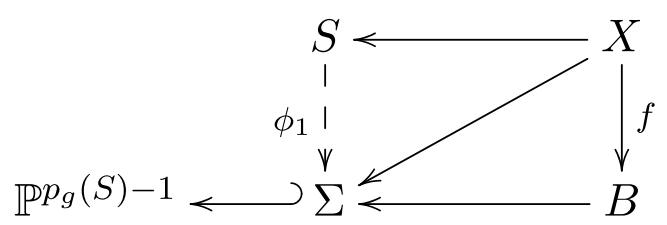

Figure 1. Canonical fibration.

\section{Preliminaries}

In this section, we recall some facts and fix the notations.

We always work over the complex number. By a surface we mean a smooth projective two-dimensional variety, and by a curve in a surface we mean a reduced one-dimensional subvariety. An integral divisor (or simply a divisor) is a finite sum $\sum n_{i} C_{i}$, where $C_{i}$ 's are irreducible curves and $n_{i} \in \mathbb{Z}$. A rational divisor is a finite sum $\sum r_{i} C_{i}$, where $C_{i}$ 's are irreducible curves and $r_{i} \in \mathbb{Q}$. A divisor $D=\sum n_{i} C_{i}$ is said to be effective, denoted by $D \geqslant 0$, if $n_{i} \geqslant 0$ for any $i$; it is said to be nef (numerically effective) if $D C \geqslant 0$ for any irreducible $C$ in the surface. Linearly and numerically equivalent relations between two divisors will be denoted by ' $\equiv$ ' and ' $\sim_{\text {num }}$ ', respectively. Similar notions apply also to the rational divisors.

Let $S$ be a smooth projective surface of general type over $\mathbb{C}$ and $K_{S}$ its canonical divisor. Denote by $p_{g}=h^{0}\left(K_{S}\right)$ the geometric genus, by $q=h^{1}\left(K_{S}\right)$ the irregularity, and by $\chi=\chi\left(\mathcal{O}_{S}\right)$ the Euler characteristic of the structure sheaf. The canonical map, denoted by $\phi_{1}$, is the map determined by the complete linear system $\left|K_{S}\right|$.

We are interested in the canonically fibered surfaces, i.e., the surfaces whose canonical map has one-dimensional image. By Stein factorization and minimal blowing up the possible base points, we obtain a relatively minimal fibration $f: X \rightarrow B$ of genus $g \geqslant 2$, which we call a canonical fibration following Sun [17], see Figure 1.

It is well known that the base $B$ is either rational or elliptic. Xiao proved in [19] that either $g(B)=q=1$, or $g(B)=0$ and $q \leqslant 2$. Let $Z$ be the fixed part of $\left|K_{X}\right|$, and $F$ be a general fiber of $f$. Then there exists an effective divisor $Z$ on $X$, and a line bundle $\mathcal{L}$ on $B$ with $p_{g}=h^{0}(\mathcal{L})$, such that

$$
K_{X}=Z+f^{*}(\mathcal{L}) \sim_{\text {num }} Z+\left(p_{g}-1+g(B)\right) F,
$$

and that

$$
f_{*} \mathcal{O}_{X}\left(K_{X}\right)=\mathcal{L} \oplus \mathcal{F}_{0},
$$

where $\mathcal{F}_{0}$ is a locally free sheaf on $B$ with $\operatorname{rank} \mathcal{F}_{0}=g-1$ and $h^{0}\left(\mathcal{F}_{0}\right)=0$.

When the fibration $f$ induced by the canonical map is hyperelliptic, we say $S$ has a hyperelliptic canonical fibration. In this case, the hyperelliptic involution on the general fiber of $f$ realizes the surface $X$ (up to the blowing-up of the isolated fixed points of hyperelliptic involution) as a double cover of a ruled surface.

We end this section by the following observation, which will be used to prove Theorem 1.6 in $\S 4.2$. 
Lemma 2.1. Let $\rho: \widetilde{P} \rightarrow P$ be a sequence of blow-ups of a geometrical ruled surface $\varphi: P \rightarrow B$. Let $\tilde{\varphi}=\varphi \circ \rho: \widetilde{P} \rightarrow B$ be the induced fibration and $\widetilde{\Gamma} \subseteq \widetilde{P}$ a general fiber of $\tilde{\varphi}$. Then for any irreducible curve $\widetilde{C} \subseteq \widetilde{P}$ with $\alpha:=\widetilde{C} \cdot \widetilde{\Gamma}>0$, one has

$$
\begin{aligned}
h^{0}(\widetilde{C}+d \widetilde{\Gamma}) & \geqslant \chi\left(\mathcal{O}_{\widetilde{P}}\right)+(\alpha+1) d+\frac{1}{2}\left(\widetilde{C}^{2}-K_{\widetilde{P}} \cdot \widetilde{C}\right) \\
& =1-g(B)+(\alpha+1) d+\frac{1}{2}\left(\widetilde{C}^{2}-K_{\widetilde{P}} \cdot \widetilde{C}\right) .
\end{aligned}
$$

Proof. This follows directly from the Riemann-Roch theorem. In fact, since $\left(K_{\widetilde{P}}-\widetilde{C}-\right.$ $d \widetilde{\Gamma}) \cdot \widetilde{\Gamma}=-3<0$, one has

$$
h^{2}(\widetilde{C}+d \widetilde{\Gamma})=h^{0}\left(K_{\widetilde{P}}-\widetilde{C}-d \widetilde{\Gamma}\right)=0 .
$$

Hence

$$
\begin{aligned}
h^{0}(\widetilde{C}+d \widetilde{\Gamma}) & \geqslant \chi(\widetilde{C}+d \widetilde{\Gamma}) \\
& =\chi\left(\mathcal{O}_{\widetilde{P}}\right)+\frac{1}{2}\left((\widetilde{C}+d \widetilde{\Gamma})^{2}-K_{\widetilde{P}} \cdot(\widetilde{C}+d \widetilde{\Gamma})\right) \\
& =\chi\left(\mathcal{O}_{\widetilde{P}}\right)+(\alpha+1) d+\frac{1}{2}\left(\widetilde{C}^{2}-K_{\widetilde{P}} \cdot \widetilde{C}\right)
\end{aligned}
$$

This proves $(2.2)$.

\section{Non-hyperelliptic canonical fibration of genus $g=4$}

In this section, we will construct the first examples of surfaces of general type with arbitrarily large geometric genus whose canonical maps induce non-hyperelliptic fibrations of genus $g=4$.

\subsection{The construction}

In this subsection, we construct a series of surfaces $S_{n}$ fibered over $\mathbb{P}^{1}$.

Let $P=\mathbb{P}^{1} \times \mathbb{P}^{1}, D_{t}=\{t\} \times \mathbb{P}^{1} \subseteq P$ with $t=0,1, \infty$, and $\Delta \subseteq P$ be the diagonal. Let $\Psi_{0}: Y_{0} \rightarrow P$ be the cyclic triple cover of $\mathbb{P}^{1}$ branched over $D_{0}+D_{1}+D_{\infty}$. Then $Y_{0} \cong E \times \mathbb{P}^{1}$, where $E$ is the elliptic curve obtained by a cyclic triple cover $\pi_{E}: E \rightarrow \mathbb{P}^{1}$ branched over $\{0,1, \infty\}$. Let $\varphi_{0}: Y_{0} \rightarrow \mathbb{P}^{1}$ be the projection. Denote by $C_{0 t} \subseteq Y_{0}$ the inverse image of $D_{t}$ for $t \in\{0,1, \infty\}$, and by $H_{0} \subseteq Y_{0}$ the inverse image of $\Delta$. Then $C_{0 t} \cdot H_{0}=1$ for $t \in\{0,1, \infty\}$.

Let $\psi_{1}: \mathbb{P}^{1} \rightarrow \mathbb{P}^{1}$ be the cyclic triple cover branched over $\{0, \infty\}$, and $Y_{1}=Y_{0} \times_{\mathbb{P}^{1}, \psi_{1}} \mathbb{P}^{1}$ the fiber product. Let $\varphi_{1}: Y_{1} \rightarrow \mathbb{P}^{1}$ be the induced fibration. Denote by $C_{1 t} \subseteq Y_{1}$ the inverse image of $C_{0 t}$, and by $H_{1} \subseteq Y_{1}$ the inverse image of $H_{0}$. Then $C_{11}$ intersects $H_{1}$ transversely at three points, which are mapped by $\varphi_{1}$ to three different points $\left\{q_{11}, q_{12}, q_{13}\right\} \subseteq \mathbb{P}^{1}$.

Let $\psi_{2}: \mathbb{P}^{1} \rightarrow \mathbb{P}^{1}$ be the cyclic triple cover branched over $\left\{q_{12}, q_{13}\right\}$, and $Y_{2}=Y_{1} \times_{\mathbb{P}^{1}, \psi_{2}}$ $\mathbb{P}^{1}$ the fiber product. Let $\varphi_{2}: Y_{2} \rightarrow \mathbb{P}^{1}$ be the induced fibration. Denote by $C_{2 t} \subseteq Y_{2}$ the inverse image of $C_{1}$, and by $H_{2} \subseteq Y_{2}$ the inverse image of $H_{1}$. The inverse image $\psi_{2}^{-1}\left(q_{11}\right)$ consists of three points, which we denote by $\left\{q_{21}, q_{22}, q_{23}\right\}$.

Let $\psi_{3}: \mathbb{P}^{1} \rightarrow \mathbb{P}^{1}$ be the cyclic triple cover branched over $\left\{q_{22}, q_{23}\right\}$, and $Y_{3}=Y_{2} \times_{\mathbb{P}^{1}, \psi_{3}}$ $\mathbb{P}^{1}$ the fiber product. Let $\varphi_{2}: Y_{2} \rightarrow \mathbb{P}^{1}$ be the induced fibration. Denote by $C_{3 t} \subseteq Y_{3}$ the 
inverse image of $C_{2 t}$, and by $H_{3} \subseteq Y_{3}$ the inverse image of $H_{2}$. The inverse image $\psi_{2}^{-1}\left(q_{21}\right)$ consists of three points, which we denote by $\left\{q_{31}, q_{32}, q_{33}\right\}$.

Continuing this process, we obtain a sequence of surfaces $Y_{n}$ together with divisors $\left\{C_{n 0}, C_{n 1}, C_{n \infty}, H_{n}\right\} \subseteq Y_{n}$.

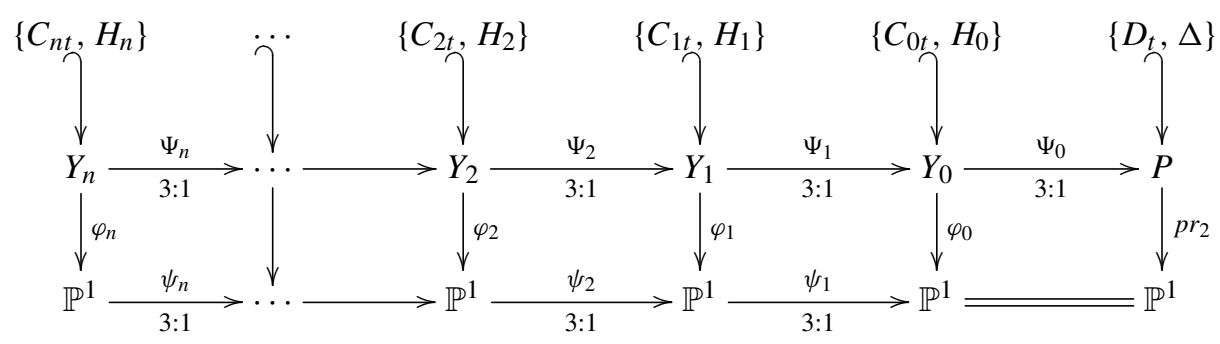

By construction,

$$
Y_{n} \cong E \times \mathbb{P}^{1}, \quad \forall n \geqslant 0 .
$$

Moreover, $C_{n t}$ is mapped to a point under the projection $Y_{n} \rightarrow E$ for $t \in\{0,1, \infty\}$. Let $\Gamma_{n} \subseteq Y_{n}$ be a general fiber of $\varphi_{n}$. Note also that the curve $E$ is just the elliptic curve given by

$$
y^{3}=x(x-1),
$$

and the cover $\pi_{E}: E \rightarrow \mathbb{P}^{1}$ is given by $(x, y) \mapsto x$. Hence for any general $x_{0} \in \mathbb{P}^{1}$, one has the following linear equivalence relation

$$
\pi_{E}^{*}\left(x_{0}\right) \equiv R_{E},
$$

where $R_{E} \subseteq E$ is the reduced ramified divisor of $\pi_{E}$. Hence for any general $x_{0} \in \mathbb{P}^{1}$,

$$
\begin{aligned}
\left(\Psi_{0} \circ \cdots \circ \Psi_{n}\right)^{*}\left(\left\{x_{0}\right\} \times \mathbb{P}^{1}\right) & =\left(\Psi_{1} \circ \cdots \circ \Psi_{n}\right)^{*}\left(\Psi_{0}^{*}\left(\left\{x_{0}\right\} \times \mathbb{P}^{1}\right)\right) \\
& \equiv\left(\Psi_{1} \circ \cdots \circ \Psi_{n}\right)^{*}\left(C_{00}+C_{01}+C_{0 \infty}\right) \\
& =C_{n 0}+C_{n 1}+C_{n \infty},
\end{aligned}
$$

and therefore,

$$
\begin{aligned}
H_{n} & =\left(\Psi_{0} \circ \cdots \circ \Psi_{n}\right)^{*}(\Delta) \\
& \equiv\left(\Psi_{0} \circ \cdots \circ \Psi_{n}\right)^{*}\left(\Lambda_{1}+\Lambda_{2}\right) \\
& =\left(\Psi_{0} \circ \cdots \circ \Psi_{n}\right)^{*}\left(\Lambda_{1}\right)+\left(\Psi_{0} \circ \cdots \circ \Psi_{n}\right)^{*}\left(\Lambda_{2}\right) \\
& =C_{n 0}+C_{n 1}+C_{n \infty}+3^{n} \Gamma_{n},
\end{aligned}
$$

where $\Lambda_{1}=\left\{x_{0}\right\} \times \mathbb{P}^{1} \subseteq P$ and $\Lambda_{2}=\mathbb{P}^{1} \times\left\{x_{0}\right\} \subseteq P$ are the two general fibers on $P$.

When $n \geqslant 1$, let $\Gamma_{n 1} \subseteq Y_{n}$ be the fiber of $\varphi_{n}$ over $q_{n 1}$. Then

$$
R_{n}=C_{n 0}+C_{n 1}+C_{n \infty}+H_{n}+\Gamma_{n 1}
$$

is 2-divisible. Hence we can construct a double cover

$$
\pi_{n}: X_{n} \rightarrow Y_{n},
$$


branched exactly over $R_{n}$. By a minimal resolution of the singularities, we obtain a smooth surface $S_{n}$. Moreover, $S_{n}$ admits a fibration $f_{n}: S_{n} \rightarrow \mathbb{P}^{1}$ induced by $\varphi_{n}$, and the genus of a general fiber of $f_{n}$ is $g=4$.

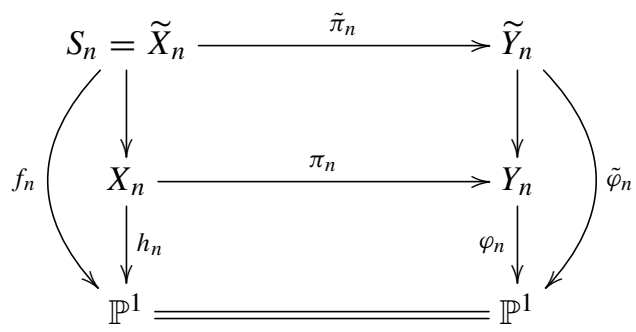

\subsection{The invariants}

In this subsection, we will prove that the surfaces $S_{n}$ 's $(n \geqslant 2)$ constructed in the last subsection admit non-hyperelliptic canonical fibrations of genus $g=4$. To this aim, we first compute the invariants of $S_{n}$ as follows.

Proposition 3.1. Let $f_{n}: S_{n} \rightarrow \mathbb{P}^{1}$ be the fibration constructed in the last subsection. Then the following statements hold.

(i)

(ii)

$$
K_{S_{n}}^{2}=4\left(3^{n}-4\right), \quad \chi\left(\mathcal{O}_{S_{n}}\right)=\frac{1}{2}\left(3^{n}-1\right) .
$$

$$
q\left(S_{n}\right)=1, \quad p_{g}\left(S_{n}\right)=\frac{1}{2}\left(3^{n}-1\right) .
$$

(iii) Let $F_{n} \subseteq S_{n}$ be a general fiber of $f_{n}$. Then $K_{S_{n}}-\frac{3^{n}-3}{2} F_{n}$ is effective and $K_{S_{n}}$ is nef if $n \geqslant 2$.

Proof. (i) As $\pi$ is a double cover branched over $R_{n}$, we may assume that $\pi_{n}$ is determined by

$$
\mathcal{O}_{Y_{n}}\left(R_{n}\right) \equiv L_{n}^{\otimes 2}
$$

for some suitable invertible sheaf $L_{n}$. To resolve the singularities of the double cover $\pi_{n}$, we perform the canonical resolution.

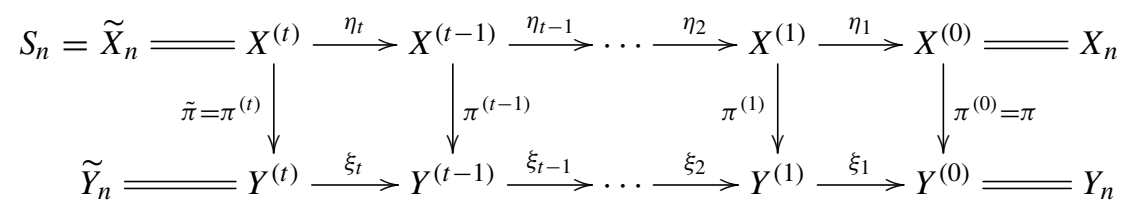

where $\xi_{i}$ 's are successive blowing-ups resolving the singularities of $R^{(0)}:=R_{n}$; and the map $\pi^{(i)}: X^{(i)} \rightarrow Y^{(i)}$ is the double cover determined by

$$
\mathcal{O}_{Y^{(i)}}\left(R^{(i)}\right) \equiv\left(L^{(i)}\right)^{\otimes 2}
$$

with

$$
\left\{\begin{array}{l}
R^{(i)}=\xi_{i}^{*}\left(R^{(i-1)}\right)-2\left[m_{i-1} / 2\right] \mathcal{E}_{i}, \\
L^{(i)}=\xi_{i}^{*}\left(L^{(i-1)}\right) \otimes \mathcal{O}_{Y^{(i)}}\left(-\left[m_{i-1} / 2\right] \mathcal{E}_{i}\right)
\end{array}\right.
$$


where $\mathcal{E}_{i} \subseteq Y^{(i)}$ is the exceptional divisor of the blowing-up $\xi_{i}, m_{i-1}$ is the multiplicity of the singular point $y_{i-1} \in R^{(i-1)}$, '[ ]' stands for the integral part, and $L^{(0)}=L_{n}$.

By the construction in the last subsection, the singularities of the branch locus $R_{n}$ are one of the following.

(1) The intersection between $H_{n}$ and $C_{n 0}: H_{n}$ intersects $C_{n 0}$ in exactly $3^{n-1}$ points, each of which is a simple singularity of $H_{n}$ of multiplicity 3 . Hence each point in $H_{n} \cap C_{n 0}$ is a simple singularity of $R_{n}$ of multiplicity 4 . In fact, locally around an intersection point of $H_{n}$ and $C_{n}$, the map $\Psi_{0} \circ \cdots \circ \Psi_{n}$ is given by

$$
u=x^{3}, \quad v=y^{3},
$$

and $H_{n}$ (resp. $C_{n 0}$ ) is given by $x^{3}-y^{3}=0$ (resp. $y=0$ ). Denote by $\mathcal{E}_{n 0} \subseteq \widetilde{Y}_{n}$ the set of exceptional curves over $H_{n} \cap C_{n 0}$. The local picture is as follows.

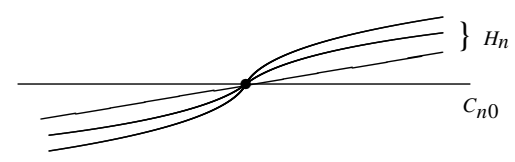

An intersection point between $H_{n}$ and $C_{n 0}$.

(2) The intersection between $H_{n}$ and $C_{n \infty}$ : this case is completely the same as the above case. That is, $H_{n} \cap C_{n \infty}$ consists also of $3^{n-1}$ points, each of which is a singularity of $R_{n}$ of multiplicity 4 . Denote by $\mathcal{E}_{n \infty} \subseteq \widetilde{Y}_{n}$ the set of exceptional curves over $H_{n} \cap C_{n \infty}$.

(3) The intersection between $H_{n}$ and $C_{n 1}$, but not over $\left\{q_{n 1}, q_{n 2}, q_{n 3}\right\}$ : there are $3^{n-1}-1$ points in $\left\{H_{n} \cap C_{n 1}\right\} \backslash\left\{\varphi^{-1}\left(q_{n 1}+q_{n 2}+q_{n 3}\right)\right\}$, each of which is again a simple singularity of $R_{n}$ of multiplicity 4 . The local situation is exactly the same as above, so we omit the local computation. Denote by $\mathcal{E}_{n 1} \subseteq \widetilde{Y}_{n}$ the set of exceptional curves over $\left\{H_{n} \cap C_{n 1}\right\} \backslash\left\{\varphi^{-1}\left(q_{n 1}+q_{n 2}+q_{n 3}\right)\right\}$.

(4) The singularities over $\left\{q_{n 1}, q_{n 2}, q_{n 3}\right\}$ : these are singularities of multiplicity of at most three, which are negligible for the double cover. In fact, locally around an intersection point of $H_{n}$ and $C_{n 1}$ over $q_{n 1}$, the map $\Psi_{0} \circ \cdots \circ \Psi_{n}$ is given by

$$
u=x, \quad v=y^{3},
$$

and $H_{n}$ (resp. $C_{n 0}$, resp. $\left.\Gamma_{n 1}\right)$ is given by $x-y^{3}=0$ (resp. $y=0$, resp. $x=0$ ). The rest singularities can be worked out similarly, and the resulted picture looks as follows.

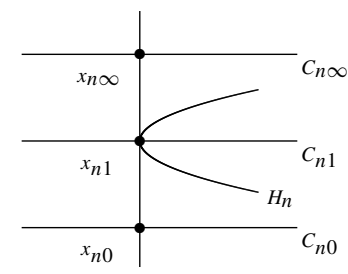

over $q_{n} 1$

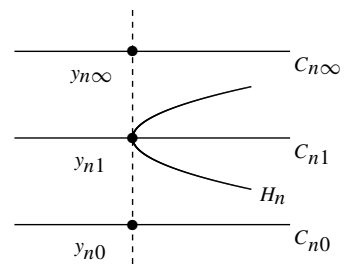

over $q_{n 2}$

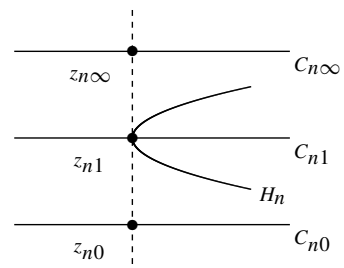

over $q_{n 3}$ 
Let $\widetilde{R}_{n}=R^{(t)} \subseteq \widetilde{Y}_{n}$ be the branch locus of $\tilde{\pi}$, and $\xi:=\xi_{1} \circ \cdots \circ \xi_{t}: \widetilde{Y}_{n} \rightarrow Y_{n}$ the composition of the blowing-ups. Then by the formulas for double covers (cf. [12, 11$]$ ), one gets that (where $\mathcal{E}=\mathcal{E}_{n 0}+\mathcal{E}_{n 1}+\mathcal{E}_{n \infty}$ )

$$
\begin{aligned}
K_{S_{n}}^{2} & =2\left(K_{\widetilde{Y}_{n}}+\frac{1}{2} \widetilde{R}_{n}\right)^{2} \\
& =2\left(\xi^{*}\left(K_{Y_{n}}+\frac{1}{2} R_{n}\right)-\mathcal{E}\right)^{2} \\
& =4\left(3^{n}-4\right) ; \\
\chi\left(\mathcal{O}_{S_{n}}\right) & =2 \chi\left(\mathcal{O}_{\widetilde{Y}_{n}}\right)+\frac{1}{4}\left(K_{\widetilde{Y}_{n}}+\frac{1}{2} \widetilde{R}_{n}\right) \cdot \widetilde{R}_{n} \\
& =2 \chi\left(\mathcal{O}_{Y_{n}}\right)+\frac{1}{4}\left(\xi^{*}\left(K_{Y_{n}}+\frac{1}{2} R_{n}\right)-\mathcal{E}\right) \cdot\left(\xi^{*}\left(R_{n}\right)-4 \mathcal{E}\right) \\
& =\frac{1}{2}\left(3^{n}-1\right) .
\end{aligned}
$$

This proves $(3.1)$.

(ii) Since $\chi\left(\mathcal{O}_{S_{n}}\right)=p_{g}\left(S_{n}\right)-q\left(S_{n}\right)+1$, it suffices to prove $q\left(S_{n}\right)=1$ in view of $(3.1)$.

Note that $Y_{n} \cong E \times \mathbb{P}^{1}$ by construction. It follows that

$$
q\left(S_{n}\right) \geqslant q\left(Y_{n}\right)=1 .
$$

On the other hand, the direct computation shows that the geometric genus of $F_{n 1}$ is $g\left(F_{n 1}\right)=1$, where $F_{n 1}$ is the fiber of $f_{n}$ over $q_{n 1}$. Hence the geometric genus of $F_{n 1}$ is $g\left(F_{n 1}\right)=1$. Thus by [8, Lemma 4.1],

$$
q\left(S_{n}\right) \leqslant g\left(F_{n 1}\right)=1 .
$$

Therefore, $q\left(S_{n}\right)=1$ as required.

(iii) Let $\mathcal{E}_{n 0}^{\prime} \subseteq \widetilde{Y}_{n}$ (resp. $\mathcal{E}_{n \infty}^{\prime} \subseteq \widetilde{Y}_{n}$ ) be the exceptional curve over $x_{n 0}$ (resp. $x_{n \infty}$ ), and $\mathcal{E}_{n 1}^{\prime} \subseteq \widetilde{Y}_{n}$ be the union of the exceptional curves over $\left\{x_{n 1}, y_{n 1}, z_{n 1}\right\}$. Denote by $\bar{C}_{n t} \subseteq \widetilde{Y}_{n}$ the strict transform of $C_{n t}$ for $t \in\{0,1, \infty\}$. Then by construction, we have

$$
\bar{C}_{n t}=\xi^{*}\left(C_{n t}\right)-\mathcal{E}_{n t}-\mathcal{E}_{n t}^{\prime}, \quad \forall t \in\{0,1, \infty\} .
$$

Note also that

$$
K_{Y_{n}}+\frac{1}{2} R_{n} \equiv C_{n 0}+C_{n 1}+C_{n \infty}+\frac{3^{n}-3}{2} \Gamma_{n} .
$$

Let $\mathcal{E}=\mathcal{E}_{n 0}+\mathcal{E}_{n 1}+\mathcal{E}_{n \infty}, \mathcal{E}^{\prime}=\mathcal{E}_{n 0}^{\prime}+\mathcal{E}_{n 1}^{\prime}+\mathcal{E}_{n \infty}^{\prime}$, and $\widetilde{\Gamma}_{n} \subseteq \widetilde{Y}_{n}$ be a general fiber of $\tilde{\varphi}$. Then

$$
\begin{aligned}
K_{S_{n}} & =\tilde{\pi}_{n}^{*}\left(K_{\widetilde{Y}_{n}}+\frac{1}{2} \widetilde{R}_{n}\right) \\
& =\tilde{\pi}_{n}^{*}\left(\xi^{*}\left(K_{Y_{n}}+\frac{1}{2} R_{n}\right)-\mathcal{E}\right) \\
& =\tilde{\pi}_{n}^{*}\left(\xi^{*}\left(C_{n 0}+C_{n 1}+C_{n \infty}+\frac{3^{n}-3}{2} \Gamma_{n}\right)-\mathcal{E}\right) \\
& =\tilde{\pi}_{n}^{*}\left(\frac{3^{n}-3}{2} \widetilde{\Gamma}_{n}+\bar{C}_{n 0}+\bar{C}_{n 1}+\bar{C}_{n \infty}+\mathcal{E}^{\prime}\right) \\
& =\frac{3^{n}-3}{2} F_{n}+\tilde{\pi}_{n}^{*}\left(\bar{C}_{n 0}+\bar{C}_{n 1}+\bar{C}_{n \infty}+\mathcal{E}^{\prime}\right) .
\end{aligned}
$$


Hence $K_{S_{n}}-\frac{3^{n}-3}{2} F_{n}$ is effective as required. Moreover, one verifies easily that there is no $(-1)$-curve contained in $\tilde{\pi}_{n}^{*}\left(\bar{C}_{n 0}+\bar{C}_{n 1}+\bar{C}_{n \infty}+\mathcal{E}^{\prime}\right)$ if $n \geqslant 2$. Therefore, $K_{S_{n}}$ is nef if $n \geqslant 2$; this is because any possible $(-1)$-curve of $S_{n}$ would be contained in the fixed part of $\left|K_{S_{n}}\right|$, and hence in $\tilde{\pi}_{n}^{*}\left(\bar{C}_{n 0}+\bar{C}_{n 1}+\bar{C}_{n \infty}+\mathcal{E}^{\prime}\right)$. This completes the proof.

The above proof shows that for $n \geqslant 2$,

$$
K_{S_{n}}=\frac{3^{n}-3}{2} F_{n}+\tilde{\pi}_{n}^{*}\left(\bar{C}_{n 0}+\bar{C}_{n 1}+\bar{C}_{n \infty}+\mathcal{E}^{\prime}\right) .
$$

Together with (3.2) one sees that $\tilde{\pi}_{n}^{*}\left(\bar{C}_{n 0}+\bar{C}_{n 1}+\bar{C}_{n \infty}+\mathcal{E}^{\prime}\right)$ is the fixed part and $\frac{3^{n}-3}{2} F_{n}$ is the movable one of the linear system $\left|K_{S_{n}}\right|$. Hence it follows that

Theorem 3.2. If $n \geqslant 2$, then the surface $S_{n}$ admits a non-hyperelliptic canonical fibration of genus $g=4$.

Remark 3.3. In our construction, the choice of the fiber $\Gamma_{n 1}$ is not essential. More precisely, we construct the double cover of $Y_{n}$ branched over $C_{n 0}+C_{n 1}+C_{n \infty}+H_{n}+\Gamma_{n 1}$. If we replace $\Gamma_{n 1}$ by $\Gamma_{n}$, where $\Gamma_{n}$ is any fiber of $\varphi_{n}$, then we can also construct a double cover of $Y_{n}$ branched over $C_{n 0}+C_{n 1}+C_{n \infty}+H_{n}+\Gamma_{n}$, and the resulted smooth surface $S_{n}^{\prime}$ (by resolution of the singularities) admits also a non-hyperelliptic canonical fibration of genus $g=4$. In fact, if $\Gamma_{n}$ is general, then the intersection between $\Gamma_{n}$ and $C_{n 0}+C_{n 1}+C_{n \infty}+H_{n}$ is again negligible. The same computation applies without any change to $S_{n}^{\prime}$. When it happens that $\Gamma_{n}$ passing though a singular point of $C_{n 0}+C_{n 1}+C_{n \infty}+H_{n}$, one can still show that $S_{n}^{\prime}$ admits a non-hyperelliptic canonical fibration of genus $g=4$ by a further careful study of the canonical resolution. We leave the details to the interested readers.

\section{Hyperelliptic canonical fibration}

In this section, we are going to exclude the existence of surfaces of general type with a hyperelliptic canonical fibration of genus $g \geqslant 4$. Hence we assume in this section that $S$ is a surface of general type which admits a hyperelliptic canonical fibration of genus $g$. Let $f: X \rightarrow B$ be the induced hyperelliptic fibration as in Figure 1. As remarked in $\S 1$, the technique differs in the elliptic and rational cases. We deal with in $\S 4.1$ the elliptic case, and in $\S 4.2$ the rational case.

\subsection{The case of elliptic base}

In this subsection, we consider the case when the base $B$ is elliptic, and prove Theorem 1.5.

Before going to the proof, we prove a general property relating to the triviality of the unitary part contained in the Hodge bundle for a hyperelliptic fibration. The idea of the proof goes back to [9].

Proposition 4.1. Let $f: X \rightarrow B$ be any non-trivial hyperelliptic fibration of $g \geqslant 2$, and $\omega_{X / B}=\mathcal{O}_{X}\left(K_{X}-f^{*} K_{B}\right)$ the relative canonical sheaf. Assume that

$$
f_{*} \omega_{X / B}=\mathcal{A} \oplus \mathcal{F}
$$


is the decomposition of the Hodge bundle $f_{*} \omega_{X / B}$ into its ample and unitary part (cf. $[4,7])$. Then after a suitable finite étale base change, $\mathcal{F}$ becomes trivial, i.e.,

$$
\mathcal{F} \cong \mathcal{O}_{B}^{\oplus r}, \quad \text { where } r=\operatorname{rank} \mathcal{F} .
$$

Proof. To prove the statement, we may assume that $\mathcal{F} \neq 0$. By construction, $\mathcal{F}$ corresponds to a unitary representation of the fundamental group

$$
\rho: \pi_{1}(B) \longrightarrow U(r) .
$$

If the image of $\rho$ is finite, then using the quotient $\pi_{1}(B) \rightarrow \operatorname{Im}(\rho)$, one can construct a finite étale cover $\widetilde{B} \rightarrow B$. Moreover, after this base change, the pull-back of $\mathcal{F}$ corresponds to the trivial representation. In other word, $\mathcal{F}$ becomes trivial and we are done. Therefore, it suffices to derive a contradiction if $\rho$ has infinite image.

By the stable reduction theorem (cf. $[1,6]$ ), there exists a base change $\phi: \widetilde{B} \rightarrow B$ of finite degree, possibly ramified, such that the pull-back fibration $\tilde{f}: \widetilde{X} \rightarrow \widetilde{B}$ is semi-stable. According to [9, Theorem A.1], applying possibly a further base change, we may assume that

$$
\tilde{f}_{*} \omega_{\widetilde{X} / \widetilde{B}}=\tilde{\mathcal{A}} \oplus \mathcal{O}_{\widetilde{B}}^{\oplus \tilde{r}}, \quad \text { where } \tilde{\mathcal{A}} \text { is ample. }
$$

Here we recall that the pull-back fibration $\tilde{f}: \widetilde{X} \rightarrow \widetilde{B}$ is constructed as follows. Let $X_{1}$ be the resolution of singularities of $X \times_{B} \widetilde{B}$. Then $\tilde{f}: \widetilde{X} \rightarrow \widetilde{B}$ is just the relatively minimal model of $X_{1}$.

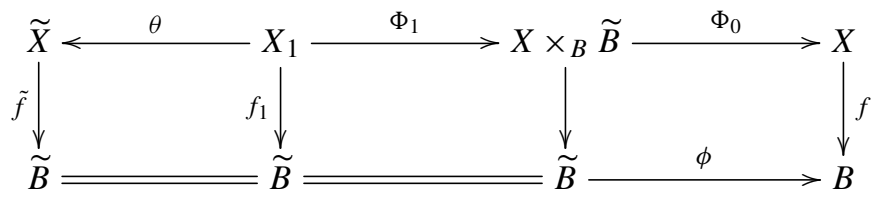

Note that there is an inclusion $\tilde{f}_{*} \omega_{\widetilde{X} / \widetilde{B}} \subseteq \phi^{*} f_{*} \omega_{X / B}$ (cf. [18, p. 231]). As

$$
\operatorname{rank} \tilde{f}_{*} \omega_{\widetilde{X} / \widetilde{B}}=\operatorname{rank} \phi^{*} f_{*} \omega_{X / B}=g,
$$

the quotient $Q:=\left(\phi^{*} f_{*} \omega_{X / B}\right) /\left(\tilde{f}_{*} \omega_{\widetilde{X} / \widetilde{B}}\right)$ is a torsion sheaf. Hence one gets a morphism:

$$
p: \tilde{f}_{*} \omega_{\widetilde{X} / \widetilde{B}} \longrightarrow \phi^{*} \mathcal{F} .
$$

It follows that the quotient $Q:=\left(\phi^{*} \mathcal{F}\right) / p\left(\tilde{f}_{*} \omega_{\widetilde{X} / \widetilde{B}}\right)$ is also a torsion sheaf. Note that $\operatorname{deg}\left(p\left(\tilde{f}_{*} \omega_{\widetilde{X} / \widetilde{B}}\right)\right) \geqslant 0$, since it is a quotient of the Hodge bundle $\tilde{f}_{*} \omega_{\widetilde{X} / \widetilde{B}}$. Note that

$$
0=\operatorname{deg} \phi^{*} \mathcal{F}=\operatorname{length}(Q)+\operatorname{deg}\left(p\left(\tilde{f}_{*} \omega_{\widetilde{X} / \widetilde{B}}\right)\right) .
$$

Hence $Q$ is zero, and the morphism $p$ is surjective.

By construction, $\phi^{*} \mathcal{F}$ comes from the following unitary representation

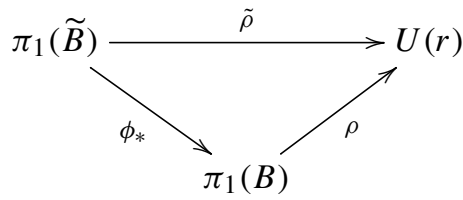


If $\rho$ has infinite image, then $\tilde{\rho}$ has also infinite image since $\phi_{*}\left(\pi_{1}(\widetilde{B})\right)$ has finite index in $\pi_{1}(B)$. Since $\widetilde{\mathcal{A}}$ in $(4.2)$ is ample, it maps to zero by $p$. Therefore we have a surjective morphism:

$$
p: \mathcal{O}_{\widetilde{B}}^{\oplus q_{\tilde{f}}} \longrightarrow \phi^{*} \mathcal{F} .
$$

Note that the trivial bundle $\mathcal{O}_{\widetilde{B}}^{\oplus q_{\tilde{f}}}$ corresponds to the trivial representation (hence also unitary representation) of $\pi_{1}(\widetilde{B})$. Hence by [11], $\phi^{*} \mathcal{F}$ is a direct summand of $\mathcal{O}_{\widetilde{B}}^{\oplus q_{\tilde{f}}}$, which implies that the representation $\tilde{\rho}$ corresponding to $\phi^{*} \mathcal{F}$ is also trivial. This gives a contradiction.

Proof of Theorem 1.5. We first show that $g \leqslant 3$. In fact, since $g(B)=1, \omega_{X / B}=$ $\mathcal{O}_{X}\left(K_{X}\right)$. By the Riemann-Roch theorem together with the semi-positivity of $f_{*} \omega_{X / B}$, one sees that the locally free sheaf $\mathcal{F}_{0}$ appearing in (2.1) is semi-stable of degree zero. Combining this with the decomposition (4.1), it follows that $\mathcal{F}_{0}$ is a unitary subsheaf. According to Proposition 4.1, after a suitable étale base change $\phi: \widetilde{B} \rightarrow B$, the pull-back fibration $\tilde{f}: \widetilde{X} \rightarrow \widetilde{B}$ satisfies

$$
\tilde{f}_{*} \omega_{\widetilde{X} / \widetilde{B}}=\mathcal{L} \oplus \mathcal{O}_{B}^{\oplus(g-1)}, \quad \text { for some ample line bundle } \mathcal{L} .
$$

In other word, the relative irregularity $q_{\tilde{f}}:=q(\widetilde{X})-g(\widetilde{B})=g-1$. As the base change is étale and $f$ is non-trivial, $\tilde{f}$ is again a non-trivial fibration. Thus by [22, Theorem 1] (see also [9, Proposition 3.2]), one gets $g-1=q_{\tilde{f}} \leqslant \frac{g+1}{2}$, which shows that $g \leqslant 3$.

Let $\tilde{f}$ be the pull-back fibration above. Since the base change is étale, it follows that $g(\widetilde{B})=g(B)=1$ and that

$$
\begin{aligned}
\omega_{\widetilde{X} / \widetilde{B}}^{2} & =K_{\widetilde{X}}^{2}=\operatorname{deg}(\phi) \cdot K_{X}^{2}, \\
\operatorname{deg} f_{*} \omega_{\widetilde{X} / \widetilde{B}} & =\chi\left(\mathcal{O}_{\widetilde{X}}\right)=\operatorname{deg}(\phi) \cdot \chi\left(\mathcal{O}_{X}\right) .
\end{aligned}
$$

By [9, Theorem 1.4], one has the slope inequality

$$
\omega_{\widetilde{X} / \widetilde{B}}^{2} \geqslant \frac{4(g-1)}{g-q_{\tilde{f}}} \cdot \operatorname{deg} f_{*} \omega_{\widetilde{X} / \widetilde{B}}=4(g-1) \operatorname{deg} f_{*} \omega_{\widetilde{X} / \widetilde{B}} .
$$

Combining these together, we obtain that

$$
K_{S}^{2}=K_{X}^{2} \geqslant 4(g-1) \chi\left(\mathcal{O}_{X}\right)
$$

Since $q=g(B)=1$ by [19], $\chi\left(\mathcal{O}_{X}\right)=\chi\left(\mathcal{O}_{S}\right)=p_{g}$. Hence we complete the proof.

\subsection{The case of rational base}

4.2.1. Technical lemmas. Let $\sigma$ be the hyperelliptic involution on $X$. Let $\epsilon: \widetilde{X} \rightarrow X$ be the blowing-up at the isolated fixed points of $\sigma$. Then $\sigma$ lifts to an involution on $\widetilde{X}$, and still denoted by $\sigma$ by abuse of notation. By construction, the quotient surface $\widetilde{P}=\widetilde{X} /\langle\sigma\rangle$ is smooth and ruled over $\mathbb{P}^{1}$. Let $\rho: \widetilde{P} \rightarrow P$ be a contraction of $\widetilde{P}$ to a geometrical ruled surface.

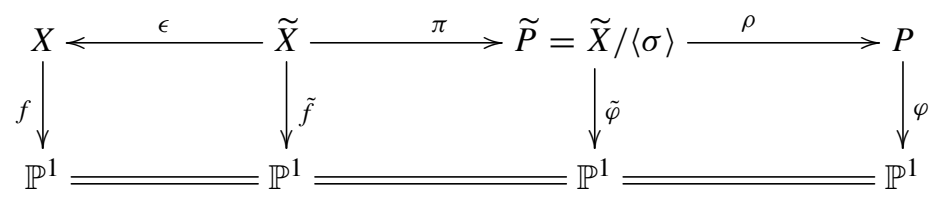


Let $F$ (resp. $\widetilde{F}$ ) be a general fiber of $f$ (resp. $\tilde{f}$ ), and $\widetilde{\Gamma}$ (resp. $\Gamma$ ) its image in $\widetilde{P}$ (resp. $P)$. Let $\sum_{j=1}^{k} \mathcal{E}_{j} \subseteq \widetilde{X}$ be the union of the exceptional curves of $\epsilon$, and

$$
K_{\tilde{X}}=\widetilde{Z}+\left(p_{g}-1\right) \widetilde{F}, \quad K_{X}=Z+\left(p_{g}-1\right) F .
$$

Here $\widetilde{Z}$ and $Z$ are the fixed parts of $\left|K_{\tilde{X}}\right|$ and $\left|K_{X}\right|$, respectively, and

$$
\widetilde{Z}=\epsilon^{*}(Z)+\sum_{j=1}^{k} \mathcal{E}_{j}
$$

Lemma 4.2. Let $C \subseteq \widetilde{Z}$ be any irreducible curve fixed by $\sigma$, i.e., $\sigma(p)=p$ for any $p \in C$. Then the multiplicity of $C$ in $\widetilde{Z}$ is even.

Proof. Let $n$ be the multiplicity of $C$ in $\widetilde{Z}$ and $p \in C$ be a general point. As $C$ is fixed by $\sigma$ by assumption, we may assume that (analytically) locally around $p, C$ is given by $y=0$, and the action of $\sigma$ is given by

$$
\sigma(x)=x, \quad \sigma(y)=-y .
$$

Take a general element $\omega \in H^{0}\left(K_{\widetilde{X}}\right)$. Then locally around $p$, we can write

$$
\omega=y^{n} h(x, y) d x \wedge d y, \quad \text { such that } h(0,0) \neq 0 .
$$

Moreover, locally around $p$, one has

$$
\sigma^{*} \omega=-(-y)^{n} h(x,-y) d x \wedge d y .
$$

Since $p_{g}(\widetilde{P})=0$, the induced action of $\sigma$ on $H^{0}\left(K_{\tilde{X}}\right)$ is just the multiplication by -1 . Hence $\sigma^{*} \omega=-\omega$, which implies that

$$
y^{n} h(x, y)=(-y)^{n} h(x,-y) .
$$

Therefore $n$ is even, since $h(0,0) \neq 0$.

Corollary 4.3. There exists an effective divisor $\widetilde{Z}^{\prime} \subseteq \widetilde{P}$ (resp. effective $\mathbb{Q}$-divisor $Z^{\prime} \subseteq \widetilde{P}$ ) such that $\widetilde{Z}=\pi^{*}\left(\widetilde{Z}^{\prime}\right)$ (resp. $\left.\epsilon^{*}(Z)=\pi^{*}\left(Z^{\prime}\right)\right)$.

Proof. It is clear that $\widetilde{Z}$ is $\sigma$-invariant. Hence it can be decomposed into sum of $\sigma$-irreducible curves:

$$
\widetilde{Z}=\sum m_{i} Z_{i}
$$

Let $Z_{i}^{\prime}=\pi\left(Z_{i}\right)$. Then

$$
\begin{cases}\pi^{*}\left(Z_{i}^{\prime}\right)=2 Z_{i} & \text { if } Z_{i} \text { is fixed by } \sigma, \\ \pi^{*}\left(Z_{i}^{\prime}\right)=Z_{i} & \text { otherwise. }\end{cases}
$$

By Lemma $4.2, m_{i}$ is even if $Z_{i}$ is fixed by $\sigma$. Hence

$$
\widetilde{Z}=\pi^{*}\left(\widetilde{Z}^{\prime}\right), \quad \text { where } \widetilde{Z}^{\prime}:=\sum m_{i} Z_{i}^{\prime}-\sum_{Z_{i} \text { fixed by } \sigma} \frac{m_{i} Z_{i}^{\prime}}{2} \geqslant 0 .
$$


Let $\sum_{j=1}^{k} \mathcal{E}_{j} \subseteq \widetilde{X}$ be the union of the exceptional curves of $\epsilon$, and $\sum_{j=1}^{k} \mathcal{E}_{j}^{\prime} \subseteq \widetilde{P}$ their images in $\widetilde{P}$. By construction, $\sum_{j=1}^{k} \mathcal{E}_{j}$ are contained in $\widetilde{Z}$ and fixed by $\sigma$. Hence

$$
Z^{\prime}=\widetilde{Z}^{\prime}-\frac{1}{2} \sum_{j=1}^{k} \mathcal{E}_{j}^{\prime}
$$

is an effective $\mathbb{Q}$-divisor, and satisfies that

$$
\pi^{*}\left(Z^{\prime}\right)=\pi^{*}\left(\widetilde{Z}^{\prime}\right)-\sum_{j=1}^{k} \mathcal{E}_{j}=\epsilon^{*}(Z) .
$$

This completes the proof.

Let

$$
K_{X}=Z+\left(p_{g}-1\right) F=D+V+\left(p_{g}-1\right) F,
$$

where $D$ (resp. $V$ ) is the horizontal (resp. vertical) part of the fixed part $Z$. In the rest part of this subsection, we would like to derive a lower bound on the canonical degree of $D$, i.e., the intersection $K_{X} D$. We introduce the following notion.

Definition 4.4. A curve $C \subseteq X$ (or $C \subseteq \widetilde{X}$ ) is said to be $\sigma$-invariant (resp. $\sigma$-fixed; resp. $\sigma$-irreducible) if $\sigma(C) \subseteq C$ (resp. if $\sigma(p)=p$ for any $p \in C$; resp. if there is an irreducible curve $C_{0}$ such that either $C=C_{0}=\sigma\left(C_{0}\right)$ or $\left.C=C_{0}+\sigma\left(C_{0}\right)\right)$.

It is clear that both $D$ and $V$ in (4.3) are $\sigma$-invariant. In particular, $D$ can be decomposed into sum of $\sigma$-irreducible curves:

$$
D=\sum_{i=1}^{t} n_{i} D_{i}
$$

where each $D_{i}$ is $\sigma$-irreducible. Denote

$$
\beta_{i}=D_{i} \cdot F
$$

Then

$$
\sum_{i=1}^{t} n_{i} \beta_{i}=2 g-2 .
$$

We arrange the indices such that $D_{i}$ is $\sigma$-fixed for $1 \leqslant i \leqslant t_{0}$, and $D_{i}$ is not $\sigma$-fixed for $t_{0}+1 \leqslant i \leqslant t$. For each $1 \leqslant i \leqslant t$, let $\widetilde{D}_{i} \subseteq \widetilde{X}$ be the strict transform of $D_{i}$, and $\widetilde{C}_{i} \subseteq \widetilde{P}$ be its image. The $\sigma$-irreducibility of $D_{i}$ implies that $\widetilde{C}_{i}$ is an irreducible curve for each $1 \leqslant i \leqslant t$.

Lemma 4.5. For $1 \leqslant i \leqslant t_{0}$, one has

$$
K_{X} D_{i} \geqslant \frac{\beta_{i}}{2}\left(p_{g}-1\right)+\frac{3}{4}\left(K_{X}+D_{i}\right) D_{i}=\frac{\beta_{i}}{2}\left(p_{g}-1\right)+\frac{3}{4}\left(K_{\widetilde{P}}+\widetilde{C}_{i}\right) \widetilde{C}_{i} .
$$


Proof. Since $D_{i}$ is $\sigma$-fixed, it follows that $D_{i} \cong \widetilde{D}_{i} \cong \widetilde{C}_{i}$ and

$$
\epsilon^{*}\left(D_{i}\right)=\widetilde{D}_{i}, \quad \pi^{*}\left(\widetilde{C}_{i}\right)=2 \widetilde{D}_{i} .
$$

Together with the adjunction formulas, one obtains

$$
K_{X} D_{i}=-D_{i}^{2}+\left(K_{\widetilde{P}}+\widetilde{C}_{i}\right) \widetilde{C}_{i}=\frac{\widetilde{C}_{i}^{2}}{2}+K_{\widetilde{P}} \widetilde{C}_{i} .
$$

By Corollary 4.3 and its proof, $\widetilde{C}_{i}$ is the fixed part of the linear system $\left|\widetilde{C}_{i}+\left(p_{g}-1\right) \widetilde{\Gamma}\right|$, where $\widetilde{\Gamma}$ is a general fiber of $\tilde{\varphi}$. Thus,

$$
h^{0}\left(\widetilde{C}_{i}+\left(p_{g}-1\right) \widetilde{\Gamma}\right)=p_{g}
$$

Note that $\widetilde{C}_{i} \cdot \widetilde{\Gamma}=D_{i} \cdot F$. Combining the above equality with Lemma 2.1, one obtains that

$$
K_{\widetilde{P}} \widetilde{C}_{i} \geqslant \widetilde{C}_{i}^{2}+2 \beta_{i}\left(p_{g}-1\right) .
$$

From this together with (4.8), it follows that

$$
K_{X} D_{i} \geqslant \frac{3}{2} \widetilde{C}_{i}^{2}+2 \beta_{i}\left(p_{g}-1\right)=3 D_{i}^{2}+2 \beta_{i}\left(p_{g}-1\right)
$$

Hence

$$
K_{X} D_{i} \geqslant \frac{\beta_{i}}{2}\left(p_{g}-1\right)+\frac{3}{4}\left(K_{X}+D_{i}\right) D_{i}=\frac{\beta_{i}}{2}\left(p_{g}-1\right)+\frac{3}{4}\left(K_{\widetilde{P}}+\widetilde{C}_{i}\right) \widetilde{C}_{i} .
$$

This proves (4.6).

Remark 4.6. In the case where $D_{i}$ is a section of $f$, one has $\beta_{i}=1$ and hence (4.6) becomes $K_{X} D_{i} \geqslant \frac{1}{2}\left(p_{g}-4\right)$, which has already been obtained by Sun [16].

Lemma 4.7. For $t_{0}+1 \leqslant i \leqslant t$, one has

$$
K_{X} D_{i} \geqslant \beta_{i}\left(p_{g}-1\right)+\left(K_{\widetilde{P}}+\widetilde{C}_{i}\right) \widetilde{C}_{i} .
$$

Proof. Assume that

$$
\epsilon^{*}\left(D_{i}\right)=\widetilde{D}_{i}+\sum_{j=1}^{k} b_{j} \mathcal{E}_{j},
$$

where $\mathcal{E}_{j}$ 's are the union of the exceptional curves of $\epsilon$. Since $D_{i}$ is $\sigma$-irreducible and not $\sigma$-fixed, one has $\pi^{*}\left(\widetilde{C}_{i}\right)=\widetilde{D}_{i}$. Hence

$$
2 \widetilde{C}_{i}^{2}=\widetilde{D}_{i}^{2}=D_{i}^{2}-\sum_{j=1}^{k} b_{j}^{2}
$$

Moreover, by Corollary 4.3 and its proof, $\widetilde{C}_{i}$ is the fixed part of the linear system $\mid \widetilde{C}_{i}+$ $\left(p_{g}-1\right) \widetilde{\Gamma} \mid$, where $\widetilde{\Gamma}$ is a general fiber of $\tilde{\varphi}$. Thus

$$
h^{0}\left(\widetilde{C}_{i}+\left(p_{g}-1\right) \widetilde{\Gamma}\right)=p_{g}
$$


Note that $\widetilde{C}_{i} \cdot \widetilde{\Gamma}=\frac{1}{2} D_{i} \cdot F$. Combining the above equality with Lemma 2.1 , one obtains that

$$
K_{\widetilde{P}} \widetilde{C}_{i} \geqslant \beta_{i}\left(p_{g}-1\right)+\widetilde{C}_{i}^{2}
$$

Let $\widetilde{R} \subseteq \widetilde{X}$ be the ramification divisor of $\pi$. Then by construction, $\sum_{j=1}^{k} \mathcal{E}_{j} \subseteq \widetilde{R}$ and $\widetilde{D}_{i} \nsubseteq \widetilde{R}$. Hence

$$
\begin{aligned}
\left(K_{\widetilde{X}}+\widetilde{D}_{i}\right) \widetilde{D}_{i} & =\left(\pi^{*}\left(K_{\widetilde{P}}+\widetilde{C}_{i}\right)+\widetilde{R}\right) \cdot \pi^{*}\left(\widetilde{C}_{i}\right) \\
& =2\left(K_{\widetilde{P}}+\widetilde{C}_{i}\right) \widetilde{C}_{i}+\widetilde{R} \cdot \widetilde{D}_{i} \\
& \geqslant 2\left(K_{\widetilde{P}}+\widetilde{C}_{i}\right) \widetilde{C}_{i}+\sum_{j=1}^{k} b_{j}
\end{aligned}
$$

Combining this with (4.10) and (4.11), one obtains that

$$
\begin{aligned}
K_{X} D_{i} & =-D_{i}^{2}+\left(K_{\widetilde{X}}+\widetilde{D}_{i}\right) \widetilde{D}_{i}+\sum_{j=1}^{k} b_{j}\left(b_{j}-1\right) \\
& \geqslant-D_{i}^{2}+2\left(K_{\widetilde{P}}+\widetilde{C}_{i}\right) \widetilde{C}_{i}+\sum_{j=1}^{k} b_{j}+\sum_{j=1}^{k} b_{j}\left(b_{j}-1\right) \\
& =2 K_{\widetilde{P}} \cdot \widetilde{C}_{i} \\
& \geqslant 2 \beta_{i}\left(p_{g}-1\right)+2 \widetilde{C}_{i}^{2} \\
& =2 \beta_{i}\left(p_{g}-1\right)+D_{i}^{2}-\sum_{j=1}^{k} b_{j}^{2} .
\end{aligned}
$$

Therefore,

$$
\begin{aligned}
K_{X} D_{i} & \geqslant \beta_{i}\left(p_{g}-1\right)+\frac{1}{2}\left(K_{X} D_{i}+D_{i}^{2}-\sum_{j=1}^{k} b_{j}^{2}\right) \\
& =\beta_{i}\left(p_{g}-1\right)+\frac{1}{2}\left(K_{\widetilde{X}} \widetilde{D}_{i}+\widetilde{D}_{i}^{2}+\sum_{j=1}^{k} b_{j}\left(b_{j}-1\right)-\sum_{j=1}^{k} b_{j}^{2}\right) \\
& \geqslant \beta_{i}\left(p_{g}-1\right)+\left(K_{\widetilde{P}}+\widetilde{C}_{i}\right) \widetilde{C}_{i} .
\end{aligned}
$$

We use (4.12) in the last inequality above. This proves (4.9).

4.2.2. The bound. In this subsection, we derive a lower bound on $K_{S}^{2}$ when $B=\mathbb{P}^{1}$, and complete the proof of Theorem 1.3(ii). First, we prove the following proposition.

Proposition 4.8. Assume that $p_{g} \geqslant 3$, and $\left|K_{S}\right|$ induces a hyperelliptic fibration. Then the moving part of $\left|K_{S}\right|$ has no fixed point, i.e., $X=S$ in Figure 1.

Proof. Assume that the moving part of $\left|K_{S}\right|$ has a fixed point. Then there exists a (-1)-curve $E$ contained in the fixed part of $\left|K_{X}\right|$ and it maps surjectively to $B$. If $E$ is fixed by the hyperelliptic involution $\sigma$, then by Lemma 4.5 one has

$$
-1=K_{X} \cdot E \geqslant \frac{1}{2}\left(p_{g}-1\right)-\frac{3}{2} \geqslant-\frac{1}{2},
$$


which is a contradiction. If $E$ is not fixed by the hyperelliptic involution $\sigma$, then by Lemma 4.7 one gets

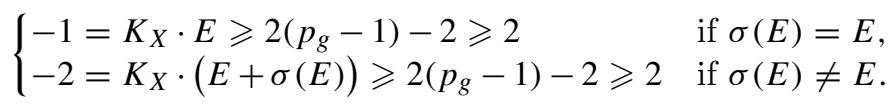

We thus get a contradiction too. This completes the proof.

We next prove a lower bound on $K_{S}^{2}$ when $B=\mathbb{P}^{1}$.

Proof of Theorem 1.6. We may assume that $p_{g} \geqslant 3$. Hence $X=S$ is already minimal by Proposition 4.8. In particular, $K_{X}$ is nef. Therefore by (4.3), (4.5) together with Lemmas 4.5 and 4.7 , we obtain

$$
\begin{aligned}
K_{S}^{2}=K_{X}^{2} \geqslant & K_{X} D+\left(p_{g}-1\right) K_{X} F \\
\geqslant & \sum_{i=1}^{t_{0}} n_{i}\left(\frac{\beta_{i}}{2}\left(p_{g}-1\right)-\frac{3}{2}\right)+\sum_{i=t_{0}+1}^{t} n_{i}\left(\beta_{i}\left(p_{g}-1\right)-2\right) \\
& +2(g-1)\left(p_{g}-1\right) \\
\geqslant & \sum_{i=1}^{t} n_{i}\left(\frac{\beta_{i}}{2}\left(p_{g}-1\right)-\frac{3}{2}\right)+2(g-1)\left(p_{g}-1\right) \\
= & 3(g-1)\left(p_{g}-1\right)-\frac{3}{2} \sum_{i=1}^{t} n_{i} \\
\geqslant & 3(g-1)\left(p_{g}-2\right) .
\end{aligned}
$$

This completes the proof.

Finally, we exclude the existence of hyperelliptic canonical fibrations of genus $g \geqslant 4$ when the geometric genus is large.

Proof of Theorem 1.3(ii). Assume that there exists a surface $S$ of general type with $p_{g}>$ $212-72 q$, which admits a hyperelliptic canonical fibration of genus $g \geqslant 4$. We will derive a contradiction.

By Theorem 1.5, the base of the induced hyperelliptic fibration is $B \cong \mathbb{P}^{1}$. Since $p_{g}>$ $212-72 q$, it follows that $p_{g} \geqslant 3$. Hence $X=S$ and $K_{X}$ is nef by Proposition 4.8. We use the notations introduced in $\S 4.2 .1$. Based on (4.3) together with Lemmas 4.5 and 4.7, we obtain that

$$
\begin{aligned}
K_{S}^{2}=K_{X}^{2} \geqslant & K_{X} D+\left(p_{g}-1\right) K_{X} F \\
\geqslant & \sum_{i=1}^{t_{0}} n_{i}\left(\frac{\beta_{i}}{2}\left(p_{g}-1\right)+\frac{3}{4}\left(K_{X}+D_{i}\right) D_{i}\right) \\
& +\sum_{i=t_{0}+1}^{t} n_{i}\left(\beta_{i}\left(p_{g}-1\right)-2\right)+2(g-1)\left(p_{g}-1\right) .
\end{aligned}
$$

Because each $D_{i}$ is fixed by the hyperelliptic involution for $1 \leqslant i \leqslant t_{0}$, it follows that every $D_{i}$ is smooth and they do not intersect each other for $1 \leqslant i \leqslant t_{0}$. Hence by applying 
the logarithmic Miyaoka-Yau inequality (cf. [10]) to the pair $\left(X, \sum_{i=1}^{t_{0}} D_{i}\right)$, one obtains that

$$
3 c_{2}\left(X \backslash \sum_{i=1}^{t_{0}} D_{i}\right) \geqslant\left(K_{X}+\sum_{i=1}^{t_{0}} D_{i}\right)^{2}
$$

Equivalently, one has

$$
K_{X}^{2} \leqslant 9 \chi\left(\mathcal{O}_{X}\right)+\sum_{i=1}^{t_{0}} \frac{\left(K_{X}+2 D_{i}\right) D_{i}}{4}=9\left(p_{g}-q+1\right)+\sum_{i=1}^{t_{0}} \frac{\left(K_{X}+2 D_{i}\right) D_{i}}{4} .
$$

Combining this together with (4.13), (4.5) and Lemma 4.5, we obtain that

$$
\begin{aligned}
& (9-3(g-1))\left(p_{g}-1\right)+18-9 q \\
& \geqslant \sum_{i=1}^{t_{0}}\left(\frac{3 n_{i}-2}{4}\left(K_{X}+D_{i}\right) D_{i}+\frac{K_{X} D_{i}}{4}\right)+\sum_{i=t_{0}+1}^{t} n_{i}\left(\frac{\beta_{i}}{2}\left(p_{g}-1\right)-2\right) \\
& \geqslant \sum_{i=1}^{t_{0}}\left(\frac{12 n_{i}-5}{16}\left(K_{X}+D_{i}\right) D_{i}+\frac{\beta_{i}}{8}\left(p_{g}-1\right)\right) \\
& \geqslant \frac{12(2 g-2)-5}{16} \cdot(-2)+\frac{1}{8}\left(p_{g}-1\right) .
\end{aligned}
$$

By rearrangement, we get

$$
p_{g} \leqslant \frac{48 g-72 q+20}{24 g-95} \leqslant 212-72 q, \quad \text { if } g \geqslant 4
$$

This contradicts the assumption.

Remarks 4.9. (i) According to the proof, if $S$ is a surface of general type admitting a hyperelliptic canonical fibration of genus $g \geqslant 4$, then the base $B \cong \mathbb{P}^{1}$, and

$$
p_{g} \leqslant \frac{48 g-72 q+20}{24 g-95} \text {. }
$$

In particular,

$$
p_{g} \leqslant \begin{cases}212-72 q & \text { if } g=4 \\ 10-\frac{72 q-10}{25} & \text { if } g=5\end{cases}
$$

(ii) X.-T. Sun points out to the author that the technique used in the case of the rational base applies also to the case of the elliptic base. In fact, applying the method here to the case of the elliptic base, one verifies without much difficulty that $g \leqslant 3$ and $K_{S}^{2} \geqslant 3(g-1) p_{g}$. However, this bound is a little weaker than that in (1.1) when $g=2$ or 3.

We end this section by constructing a sequence of hyperelliptic canonical fibrations of genus $g=3$, where the geometric genus can be arbitrarily large. In fact, one shows without difficulty that our examples are generalized-hyperelliptic surfaces, which were systematically studied by Zucconi in [25, 26]. 
Example 4.10. Let $F$ be the hyperelliptic curve of genus 3 defined by $u^{2}=v^{8}-1$, and $\tau_{1}$ be the involution of $F$ defined by $\tau_{1}(u, v)=(-u,-v)$. Then $\tau_{1}$ has no fixed point, and $\Gamma=F /\left\langle\tau_{1}\right\rangle$ is of genus 2. Let $C_{0}$ be any hyperelliptic curve of genus $g_{0} \geqslant 2$, and $\sigma_{0}$ the hyperelliptic involution. Then $\tau:=\left(\tau_{1}, \sigma_{0}\right)$ is an involution on the product $F \times C_{0}$, and admits no fixed point. Hence the quotient surface $S=F \times C_{0} /\langle\tau\rangle$ is smooth, and admits a (non-trivial) hyperelliptic fibration $f: S \rightarrow \mathbb{P}^{1}=C_{0} /\left\langle\sigma_{0}\right\rangle$. One computes easily that

$$
K_{S}^{2}=8\left(g_{0}-1\right), \quad \chi\left(\mathcal{O}_{S}\right)=g_{0}-1 .
$$

Moreover, by construction, $S$ admits another fibration $h: S \rightarrow \Gamma=F /\left\langle\tau_{1}\right\rangle$. Hence $q(S)=$ $g(\Gamma)=2$, which implies that $p_{g}(S)=\chi\left(\mathcal{O}_{S}\right)-1+q(S)=g_{0}$. It follows that

$$
f_{*} \mathcal{O}_{S}\left(K_{S}\right) \cong \mathcal{O}_{\mathbb{P}^{1}}\left(g_{0}-1\right) \oplus \mathcal{O}_{\mathbb{P}^{1}}(-2)^{\oplus 2} \text {. }
$$

Hence $S$ is a surface of general type admitting a hyperelliptic canonical fibration of genus $g=3$. Moreover, when $g_{0}$ goes to the infinity, the geometric genus of $S$ can be arbitrarily large.

\section{Acknowledgments}

The author would like to thank Prof. X.-T. Sun for his careful reading and many useful suggestions. The author appreciates the referee very much for many helpful comments for the correction of the original manuscript.

\section{References}

1. M. Artin And G. Winters, Degenerate fibres and stable reduction of curves, Topology 10 (1971), 373-383, MR 0476756.

2. A. Beauville, L'application canonique pour les surfaces de type général, Invent. Math. 55(2) (1979), 121-140, MR 553705.

3. F. Catanese, Singular bidouble covers and the construction of interesting algebraic surfaces, in Algebraic Geometry: Hirzebruch 70 (Warsaw, 1998), Contemporary Mathematics, Volume 241, pp. 97-120 (American Mathematical Society, Providence, RI, 1999), MR 1718139.

4. F. Catanese and M. Dettweiler, Answer to a question by fujita on variation of hodge structures, preprint, 2013, arXiv:1311.3232.

5. X. Chen, Xiao's conjecture on canonically fibered surfaces, Adv. Math. 322 (2017), $1033-1084$.

6. P. Deligne and D. Mumford, The irreducibility of the space of curves of given genus, Publ. Math. Inst. Hautes Études Sci. 36 (1969), 75-109, MR 0262240.

7. T. Fujita, The sheaf of relative canonical forms of a Kähler fiber space over a curve, Proc. Japan Acad. Ser. A Math. Sci. 54(7) (1978), 183-184, MR 510945 (80b:14004).

8. J. Lu, S.-L. TAN, F. YU AND K. ZuO, A new inequality on the Hodge number $h^{1,1}$ of algebraic surfaces, Math. Z. 276(1-2) (2014), 543-555, MR 3150217.

9. X. LU AND K. ZuO, On the slope of hyperelliptic fibrations with positive relative irregularity, Trans. Amer. Math. Soc. 369(2) (2017), 909-934, MR 3572259.

10. Y. MiYAoKA, The maximal number of quotient singularities on surfaces with given numerical invariants, Math. Ann. 268(2) (1984), 159-171, MR 744605. 
11. M. S. NARAsimhan AND C. S. Seshadri, Stable and unitary vector bundles on a compact Riemann surface, Ann. of Math. (2) 82 (1965), 540-567, MR 0184252.

12. U. Persson, Chern invariants of surfaces of general type, Compos. Math. 43(1) (1981), 3-58, MR 631426.

13. R. Pignatelli, On surfaces with a canonical pencil, Math. Z. 270(1-2) (2012), 403-422, MR 2875841.

14. G. PompILJ, Alcuni esempi di superficie algebriche a sistema canonico puro degenere, Atti Accad. Naz. Lincei. Rend. Cl. Sci. Fis. Mat. Natur. 84 (1948), 539-544, MR 0027562.

15. I. REIDER, Vector bundles of rank 2 and linear systems on algebraic surfaces, Ann. of Math. (2) 127(2) (1988), 309-316, MR 932299.

16. X. T. Sun, Surfaces of general type with canonical pencil, Acta Math. Sinica 33(6) (1990), 769-773, MR 1090625.

17. X. T. Sun, On canonical fibrations of algebraic surfaces, Manuscripta Math. 83(2) (1994), 161-169, MR 1272180.

18. S. L. TAN, On the invariants of base changes of pencils of curves. I, Manuscripta Math. 84(3-4) (1994), 225-244, MR 1291119.

19. G. XiAO, L'irrégularité des surfaces de type général dont le système canonique est composé d'un pinceau, Compos. Math. 56(2) (1985), 251-257, MR 809870.

20. G. XIAO, Surfaces fibrées en courbes de genre deux, Lecture Notes in Mathematics, Volume 1137 (Springer, Berlin, 1985), MR 872271.

21. G. XIAO, Problem List, in Birational Geometry of Algebraic Varities: Open Problems, The XXIIIrd International Symposium of the Taniguchi Foundation, 1988.

22. G. XıAO, Irregular families of hyperelliptic curves, in Algebraic geometry and algebraic number theory (Tianjin, 1989-1990), Nankai Ser. Pure Appl. Math. Theoret. Phys., Volume 3, pp. 152-156 (World Sci. Publ., River Edge, NJ, 1992), MR 1301093.

23. J.-G. YANG AND M. MiYANISHI, Surfaces of general type whose canonical map is composed of a pencil of genus 3 with small invariants, J. Math. Kyoto Univ. 38(1) (1998), 123-149, MR 1628083.

24. F. ZUCCONI, Numerical inequalities for surfaces with canonical map composed with a pencil, Indag. Math. (N.S.) 9(3) (1998), 459-476, MR 1692141.

25. F. ZuCCONI, Abelian covers and isotrivial canonical fibrations, Comm. Algebra 29(12) (2001), 5641-5671, MR 1872817.

26. F. Zucconi, Generalized hyperelliptic surfaces, Trans. Amer. Math. Soc. 355(10) (2003), 4045-4059, MR 1990574. 